\title{
Rigidity and Normal Modes in Random Matrix Spectra
}

\author{
A. Andersen, A. D. Jackson, and H. J. Pedersen \\ The Niels Bohr Institute, Blegdamsvej 17, DK-2100 Copenhagen Ø, Denmark
}

August 8, 2018

\begin{abstract}
We consider the Gaussian ensembles of random matrices and describe the normal modes of the eigenvalue spectrum, i.e., the correlated fluctuations of eigenvalues about their most probable values. The associated normal mode spectrum is linear, and for large matrices, the normal modes are found to be Chebyshev polynomials of the second kind. We contrast this with the behaviour of a sequence of uncorrelated levels, which has a quadratic normal mode spectrum. The difference in the rigidity of random matrix spectra and sequences of uncorrelated levels can be attributed to this difference in the normal mode spectra. We illustrate this by calculating the number variance in the two cases.
\end{abstract}

\section{Introduction}

Random matrices have been used to describe level correlations in many different areas of physics from nuclear levels to acoustic resonances to QCD. See, e.g., the review [1]. The most important feature distinguishing the eigenvalues of a random matrix from a sequence of uncorrelated levels is the existence of strong repulsion between neighbouring eigenvalues. This repulsion is conveniently pictured by the Coulomb gas model adopted by Dyson in 1962 [2]. Dyson exploited the equivalence between the Gaussian ensembles of random matrices and a classical problem in electrostatics in which identical uniformly charged parallel wires are placed on a line in a harmonic confining external field. The eigenvalues of the random matrix experience a repulsion which is analogous to the Coulomb repulsion between the line charges. This level repulsion leads to the rigidity 
of random matrix spectra, which is most easily illustrated by the fact that the variance of the number of eigenvalues in an (unfolded) interval of length $L$ grows logarithmically with $L$. This is in contrast to the linear behaviour of the number variance for a sequence of uncorrelated levels.

All spectral properties of random matrices can be calculated from the joint probability distribution of the eigenvalues. Given the Coulomb analogy, it is natural to consider the independent "normal modes" of the joint probability density, which describe the correlated motion of eigenvalues about their most probable values. Evidently, these normal modes have a simple interpretation in the Coulomb analogy where they describe the independent oscillations of charges on a lattice about their equilibrium positions. An eigenvalue can be associated with each normal mode, and these eigenvalues form the normal mode spectrum. In the present case, soft (hard) modes correspond to large (small) amplitude fluctuations in the random matrix spectrum. These collective degrees of freedom prove to be useful in determining the long-range spectral fluctuation measures of random matrices including the number variance mentioned above.

The purpose of this paper is to calculate the normal modes of the Gaussian random matrix ensembles and to use them for a determination of the number variance. For comparison, we will also determine the corresponding normal modes and the number variance for a sequence of uncorrelated levels. We emphasise that the results of this exercise are neither new nor exact. Our intention is rather to offer a new way to regard the fluctuations in random matrix spectra and to emphasise the value of thinking about the correlated motion of eigenvalues.

\section{Normal Modes for the Gaussian Ensembles}

The Gaussian ensembles of $N \times N$ matrices have a joint probability distribution of the eigenvalues $x_{1}, x_{2}, \ldots, x_{N}$ which is [3]

$$
P_{N \beta}\left(x_{1}, x_{2}, \ldots, x_{N}\right)=C_{N \beta} \prod_{1 \leq i<j \leq N}\left|x_{i}-x_{j}\right|^{\beta} \exp \left(-\frac{\beta}{2} N \sum_{i=1}^{N} x_{i}^{2}\right),
$$

where $\beta=1,2,4$ corresponds to the Gaussian orthogonal, unitary, and symplectic ensembles, respectively. This distribution leads to an average level density $\rho(x)$ which is independent of $\beta$. In the large- $N$ limit:

$$
\rho(x)=\frac{N}{\pi} \sqrt{2-x^{2}} .
$$


The maxima of $P_{N \beta}$ correspond to the most probable locations of the eigenvalues. It is sufficient to consider the single maximum with $x_{i}<x_{i+1}$, and we denote the maximum value of $P_{N \beta}$ by $P_{N \beta}^{0}$. One immediately obtains the following set of equations which determine the equilibrium positions of the eigenvalues

$$
\sum_{j \neq i} \frac{1}{x_{i}-x_{j}}-N x_{i}=0 .
$$

In the vicinity of the maximum, we approximate the logarithm of $P_{N \beta}$ by

$$
\ln P_{N \beta}=\ln P_{N \beta}^{0}+\frac{1}{2} \beta \sum_{i, j} \delta x_{i} C_{i j} \delta x_{j} .
$$

The matrix $C$ is defined as

$$
C_{i j}=\frac{1}{\beta} \frac{\partial^{2}}{\partial x_{i} \partial x_{j}} \ln P_{N \beta}
$$

evaluated at the maximum. The elements of $C$ are

$$
\begin{aligned}
C_{i i} & =-\sum_{j \neq i} \frac{1}{\left(x_{i}-x_{j}\right)^{2}}-N \\
C_{i j} & =\frac{1}{\left(x_{i}-x_{j}\right)^{2}} .
\end{aligned}
$$

The eigenvectors of $C$ are the normal modes of the random matrix spectrum. Each of these eigenvectors describes a statistically independent mode of correlated motion of the eigenvalues of the random matrix. Clearly, they provide the natural basis in which to describe the behaviour of $P_{N \beta}$ in the vicinity of its maximum.

The solution to (3) for an $N \times N$ matrix is given by the zeros of the Hermite polynomial, $H_{N}$

$$
H_{N}\left(\sqrt{N} x_{i}\right)=0
$$

This result was obtained by Stieltjes (see appendix A.6 in ref. [3]). It follows from the observation that Hermite's differential equation,

$$
H_{N}^{\prime \prime}(x)-2 x H_{N}^{\prime}(x)+2 N H_{N}(x)=0
$$

reduces to (3) at the zeros of $H_{N}$.

The results for the eigenvalues and eigenvectors of the matrix $C$ are readily stated. We prove these results in sections 3 and 4 . The eigenvalues $\lambda_{k}$ of $C$, which satisfy

$$
\sum_{j=1}^{N} C_{i j} \delta y_{j}^{(k)}=\lambda_{k} \delta y_{i}^{(k)}
$$


are remarkably simple. Namely,

$$
\lambda_{k}=-k N
$$

where $k$ runs from 1 to $N$. The $i$-th component of the corresponding eigenvector is a polynomial of order $k-1$ evaluated at the equilibrium value of $x_{i}$. The first four normalised eigenvectors can be written as

$$
\begin{aligned}
\delta y_{i}^{(1)} & =\frac{1}{N^{1 / 2}} \\
\delta y_{i}^{(2)} & =\left(\frac{2}{N-1}\right)^{1 / 2} x_{i} \\
\delta y_{i}^{(3)} & =\left(\frac{N-1}{N(N-2)}\right)^{1 / 2}\left(1-\frac{2 N}{N-1} x_{i}^{2}\right) \\
\delta y_{i}^{(4)} & =\left(\frac{2(2 N-3)^{2}}{(N-1)(N-2)(N-3)}\right)^{1 / 2}\left(x_{i}-\frac{2 N}{2 N-3} x_{i}^{3}\right) .
\end{aligned}
$$

In the large- $N$ limit, the eigenvector component $\delta y_{i}^{(k)}$ is given by the value of the Chebyshev polynomial of the second kind, $U_{k-1}(x)$, evaluated at the point $x=x_{i} / \sqrt{2}$. One finds that (up to corrections of order $1 / N$ )

$$
\delta y_{i}^{(k)}=N^{-1 / 2} U_{k-1}\left(\frac{x_{i}}{\sqrt{2}}\right) .
$$

This result is natural when one notices that the identity

$$
\sum_{i=1}^{N} \delta y_{i}^{(k)} \delta y_{i}^{(l)}=\delta_{k l}
$$

can be approximated for large $N$ by

$$
\int_{-\sqrt{2}}^{\sqrt{2}} \mathrm{~d} x \rho(x) \delta y^{(k)}(x) \delta y^{(l)}(x)=\delta_{k l}
$$

with $\rho(x)$ given by (2) . Eqn. (18) is seen to be the orthogonality relation for the Chebyshev polynomials of the second kind.

\section{Eigenvalues and Eigenvectors of $C$ for Finite $N$}

To prove relations (11) to (15), begin by looking at the definition of $C$ stated in (6) and (7), and let $C$ act on a power of $x_{i}$. We obtain

$$
\sum_{j=1}^{N} C_{i j} x_{j}^{k}=\sum_{j \neq i} \frac{x_{j}^{k}-x_{i}^{k}}{\left(x_{j}-x_{i}\right)^{2}}-N x_{i}^{k}
$$




$$
\begin{aligned}
& =\sum_{l=0}^{k-2}(l+1) x_{i}^{l} \sum_{j \neq i} x_{j}^{k-l-2}+k x_{i}^{k-1} \sum_{j \neq i} \frac{1}{x_{j}-x_{i}}-N x_{i}^{k} \\
& =\sum_{l=0}^{k-2}(l+1) \sigma_{k-l-2} x_{i}^{l}-\frac{1}{2} k(k-1) x_{i}^{k-2}-(k+1) N x_{i}^{k} .
\end{aligned}
$$

Here it is understood that the sums over $l$ are zero for $k \leq 1$ and that

$$
\sigma_{m} \equiv \sum_{j=1}^{N} x_{j}^{m}
$$

The second identity in (19) can be proved by induction, and eqn. (3) can be used to obtain the third identity. It follows from (19) that the $i$-th component of the $k$-th eigenvector,

$\delta y_{i}^{(k)}$, is a polynomial of order $k-1$ in $x_{i}$ and that the corresponding eigenvalue is the coefficient, $-k N$, of the highest order term, $x_{i}^{k-1}$.

The sums $\sigma_{m}$ can be determined using the relation

$$
\sum_{i=1}^{N} \sum_{j=1}^{N} C_{i j} x_{j}^{k}=-N \sum_{i=1}^{N} x_{i}^{k}=-N \sigma_{k},
$$

which, together with (19), lead to the recursion relation

$$
N k \sigma_{k}=\sum_{l=0}^{k-2}(l+1) \sigma_{k-l-2} \sigma_{l}-\frac{1}{2} k(k-1) \sigma_{k-2} .
$$

One observes directly that $\sigma_{0}=N$. Since the $x_{i}$ come in pairs $\pm x$ (or are 0 ), it follows that $\sigma_{2 k-1}=0$ for all $k \geq 1$. This fact and the recursion relation above permit the determination of all of the sums $\sigma_{k}$.

Knowing the values of the sums, $\sigma_{k}$, it is now possible to obtain all eigenvectors of $C$ using (19) and to verify directly that the vectors (12) to (15) are indeed eigenvectors of $C$ with the eigenvalues stated in (11).

\section{The Large- $N$ Limit}

In this section we show that, in the large- $N$ limit, the eigenvectors of $C$ are related to the Chebyshev polynomials of the second kind as indicated in equation (16). Define a generating function $G$ by the equation

$$
G(x) \equiv \sum_{k=0}^{\infty} \sigma_{2 k} x^{2 k},
$$


where the $\sigma_{k}$ are now defined by the large- $N$ form of equation (22) which reads

$$
N k \sigma_{k}=\sum_{l=0}^{k-2}(l+1) \sigma_{k-l-2} \sigma_{l} .
$$

Multiplication by $x^{k-1}$ and subsequent summation over $k$ leads to an equation which is equivalent to the following differential equation for $G$

$$
N \frac{\mathrm{d}}{\mathrm{d} x} G=(x G) \frac{\mathrm{d}}{\mathrm{d} x}(x G) .
$$

Since $\sigma_{0}=N$, it follows that $G(0)=N$, and that

$$
G(x)=N \frac{1-\sqrt{1-2 x^{2}}}{x^{2}} .
$$

From the definition of $G$ in expression (23), one observes that the $\sigma_{2 k}$ are simply

$$
\sigma_{2 k}=N \frac{(2 k-1) ! !}{(k+1) !}
$$

These values of $\sigma_{2 k}$ can also be obtained from the integral

$$
\sigma_{2 k}=\int_{-\sqrt{2}}^{\sqrt{2}} \mathrm{~d} x x^{2 k} \rho(x)
$$

where $\rho(x)$ is the Wigner semi-circle describing the average level density (2).

With these expressions for the sums $\sigma_{2 k}$, one can construct the eigenvectors of $C$ using a Gram-Schmidt orthogonalisation procedure starting with a non-orthogonal basis of vectors with elements $x_{i}^{k}$. The coefficients to be determined in the Gram-Schmidt procedure are

$$
a_{k j}=-\sum_{i=1}^{N} x_{i}^{k-1} \delta y_{i}^{(j)}=-\int_{-\sqrt{2}}^{\sqrt{2}} \mathrm{~d} x x^{k-1} \delta y^{(j)}(x) \rho(x) .
$$

The structure of the integrals implies that the $\delta y^{(k)}(x)$ are simply the polynomials orthogonal on the interval $[-\sqrt{2}, \sqrt{2}]$ with weight $\rho(x)$. These polynomials are recognised as the Chebyshev polynomials of the second kind, appropriately scaled. This shows that the elements of the eigenvectors $\delta y_{i}^{(k)}$ are given by (16) in the large- $N$ limit.

\section{The Number Variance for the Gaussian Ensembles}

The small amplitude, quadratic approximation to $P_{N \beta}$ in terms of its normal modes is useful in calculations of long range spectral fluctuation measures. We illustrate this by 
determining the asymptotic form of the number variance for the Gaussian ensembles. The number variance, $\Sigma^{2}(L)$, is defined as the variance of the number of eigenvalues in an interval of length $L$. (Here, it is assumed that the spectrum has been "unfolded" so that the average spacing between adjacent eigenvalues is 1.) It is well-known that the exact number variance $\Sigma_{\beta}^{2}(L)$ for the Gaussian ensembles has the form [1]

$$
\Sigma_{\beta}^{2}(L)=\frac{2}{\beta \pi^{2}} \ln L+K_{\beta}+O\left(L^{-1}\right),
$$

where $K_{\beta}$ is a constant. The leading logarithmic term in this expression is indicative of a rigid sequence of numbers. This is to be contrasted with the linear $L$-dependence which characterises the number variance for a sequence of uncorrelated levels.

We wish to reproduce the logarithmic term in the expression for the number variance using the normal modes. Consider an interval of the unfolded eigenvalue spectrum from $-L / 2$ to $L / 2$. For sufficiently large $N$, the level density of the original spectrum corresponding to this part of the unfolded spectrum has the constant value of $\rho(0)=$ $\sqrt{2} N / \pi$. Within this interval, the equilibrium position of the $k$-th eigenvalue is therefore

$$
x_{k}^{(0)}=\frac{\pi k}{\sqrt{2} N} .
$$

Fluctuations in the eigenvalue spectrum will move the $k$-th eigenvalue to a new position which can be written as

$$
x_{k}=x_{k}^{(0)}+\sum_{n=1}^{N} \alpha_{n} \delta y_{k}^{(n)}=\frac{\pi k}{\sqrt{2} N}+\frac{1}{\sqrt{N}} \sum_{n=1}^{N} \alpha_{n} U_{n-1}\left(\frac{\pi k}{2 N}\right) .
$$

This means that, when fluctuations are present, the eigenvalues at the (unfolded) energies $\pm L / 2$ will have eigenvalue numbers

$$
k_{ \pm}= \pm \frac{L}{2}-\frac{\sqrt{2 N}}{\pi} \sum_{n=1}^{N} \alpha_{n} U_{n-1}\left( \pm \frac{\pi L}{4 N}\right) .
$$

Since the number of levels in the interval is now $k_{+}-k_{-}$, it follows that the number variance can be approximated by the ensemble averages

$$
\begin{aligned}
\Sigma_{\beta}^{2}(L) & \approx\left\langle\left(k_{+}-k_{-}\right)^{2}\right\rangle-\left\langle k_{+}-k_{-}\right\rangle^{2} \\
& =\frac{8 N}{\pi^{2}} \sum_{n=1}^{\left[\frac{N}{2}\right]}\left\langle\alpha_{2 n}^{2}\right\rangle U_{2 n-1}^{2}\left(\frac{\pi L}{4 N}\right) \\
& =\frac{4}{\beta \pi^{2}} \sum_{n=1}^{\left[\frac{N}{2}\right]} \frac{1}{n} U_{2 n-1}^{2}\left(\frac{\pi L}{4 N}\right) .
\end{aligned}
$$


Here, we have made use of the fact that terms involving Chebyshev polynomials of even order cancel and that the averages over the coefficients are

$$
\left\langle\alpha_{i} \alpha_{j}\right\rangle=\frac{1}{j \beta N} \delta_{i j} .
$$

This last result can be understood by expanding the $\delta x_{i}$ in the eigenvectors of $C$,

$$
\delta x_{i}=\sum_{k=1}^{N} \alpha_{k} \delta y_{i}^{(k)} .
$$

The joint probability distribution can now be interpreted as the distribution of the $\alpha_{k}$. In the approximation (4), this distribution becomes

$$
\begin{aligned}
P_{N \beta}\left(\alpha_{1}, \alpha_{2}, \ldots, \alpha_{N}\right) & =P_{N \beta}^{0} e^{\frac{1}{2} \beta \sum_{i, j} \delta x_{i} C_{i j} \delta x_{j}} \\
& =P_{N \beta}^{0} e^{\frac{1}{2} \beta \sum_{k} \lambda_{k} \alpha_{k}^{2}} \\
& =P_{N \beta}^{0} \prod_{k} e^{\frac{1}{2} \beta \lambda_{k} \alpha_{k}^{2}},
\end{aligned}
$$

from which it is clear that the $\alpha_{k}$ are independent and Gaussian distributed with variance (35).

To calculate the sum over the Chebyshev polynomials, we introduce a new variable $\theta$ defined as $\cos \theta=\pi L / 4 N$ for $L<4 N$ and set $K \equiv[N / 2]$. The terms in the sum can be rewritten as integrals, and after an interchange of integration and summation we obtain

$$
\sum_{n=1}^{K} \frac{1}{n} U_{2 n-1}^{2}\left(\frac{\pi L}{4 N}\right)=\frac{2}{\sin ^{2} \theta} \int_{0}^{\theta} \mathrm{d} \theta^{\prime} \frac{\sin 2 K \theta^{\prime} \sin 2(K+1) \theta^{\prime}}{\sin 2 \theta^{\prime}} .
$$

The value $L=0$ corresponds to $\theta=\pi / 2$. For this value, the integral equals zero. We can now express the approximate number variance as

$$
\Sigma_{\beta}^{2}(L) \approx \frac{8}{\beta \pi^{2} \sin ^{2} \theta} \int_{0}^{\frac{\pi}{2}-\theta} \mathrm{d} \theta^{\prime} \frac{\sin 2 K \theta^{\prime} \sin 2(K+1) \theta^{\prime}}{\sin 2 \theta^{\prime}} .
$$

For fixed $L$ and large $K$, we see that

$$
\frac{\pi}{2}-\theta=\frac{\pi L}{4(2 K)}
$$

In this limit, our approximation to the number variance can be written as

$$
\Sigma_{\beta}^{2}(L) \approx \frac{4}{\beta \pi^{2}} \int_{0}^{\frac{\pi L}{4}} \mathrm{~d} x \frac{\sin ^{2} x}{x}=\frac{2}{\beta \pi^{2}}\left[\ln L-\mathrm{Ci}\left(\frac{\pi L}{2}\right)+\ln \frac{\pi}{2}+\gamma\right],
$$


where $\operatorname{Ci}(\pi L / 2)$ is the cosine integral and $\gamma$ is Eulers constant. For large values of $L$, this function can be approximated by

$$
\Sigma_{\beta}^{2}(L) \approx \frac{2}{\beta \pi^{2}}\left(\ln L+\ln \frac{\pi}{2}+\gamma\right) .
$$

This expression contains a logarithmic term identical to that in (30). The leading term in the number variance for large $L$ is thus obtained correctly by the Gaussian approximation to $P_{N \beta}$. The Gaussian approximation is not sufficient to reproduce the constant term in $(30)$.

\section{A Sequence of Uncorrelated Levels}

It is useful to find a similar description of the normal modes for a sequence of uncorrelated levels. In this case, it is easiest to proceed by considering the correlation matrix

$$
D_{i j}=\left\langle\left(x_{i}-\left\langle x_{i}\right\rangle\right)\left(x_{j}-\left\langle x_{j}\right\rangle\right)\right\rangle=\left\langle x_{i} x_{j}\right\rangle-\left\langle x_{i}\right\rangle\left\langle x_{j}\right\rangle
$$

where $\left\langle x_{k}\right\rangle$ is the ensemble average of eigenvalue $k$. This approach is slightly different from that adopted above when we considered a quadratic approximation to $\ln P_{N \beta}$. To the extent that this approximation is exact, the two approaches lead to identical eigenvectors and eigenvalues which are negative reciprocals of one another.

A spectrum of $N$ uncorrelated levels with unit mean level density has a Poisson distribution for the level spacings. The joint probability distribution for the levels can thus be written as a product of Poisson distributions and step functions:

$$
\begin{aligned}
P_{N}\left(x_{1}, x_{2}, \ldots, x_{N}\right) & =e^{-x_{1}} \theta\left(x_{1}\right) \prod_{i=1}^{N-1} e^{-\left(x_{i+1}-x_{i}\right)} \theta\left(x_{i+1}-x_{i}\right) \\
& =e^{-x_{N}} \theta\left(x_{1}\right) \prod_{i=1}^{N-1} \theta\left(x_{i+1}-x_{i}\right) .
\end{aligned}
$$

This form of the joint probability distribution leads immediately to the ensemble averages $\left\langle x_{i}\right\rangle$ and $\left\langle x_{i} x_{j}\right\rangle$ from which the elements of the matrix $D$ follow:

$$
D_{i j}=\min \{i, j\}
$$

The eigenvalues, $\omega_{k}$, and eigenvectors, $\psi_{i}^{(k)}$, of $D$ can be obtained by exploiting the special structure of $D$ and expressing the eigenvalue problem in the following suggestive 
manner:

$$
\begin{aligned}
-\psi_{1}^{(k)} & =\omega_{k}\left(\psi_{2}^{(k)}-2 \psi_{1}^{(k)}\right) \\
-\psi_{i}^{(k)} & =\omega_{k}\left(\psi_{i-1}^{(k)}-2 \psi_{i}^{(k)}+\psi_{i+1}^{(k)}\right) \\
\psi_{N}^{(k)} & =\omega_{k}\left(\psi_{N}^{(k)}-\psi_{N-1}^{(k)}\right)
\end{aligned}
$$

where $2 \leq i \leq N-1$. Introducing the definition $\phi_{k} \equiv(2 k-1) \pi /(2 N+1)$, it is readily verified that the normalised eigenvectors can be written as

$$
\psi_{j}^{(k)}=\frac{2}{\sqrt{2 N+1}} \sin \left(j \phi_{k}\right) .
$$

The corresponding eigenvalues are

$$
\omega_{k}=\frac{1}{4} \sin ^{-2}\left(\frac{\phi_{k}}{2}\right) .
$$

To facilitate comparison with the random matrix results obtained above, it is useful to multiply the $\omega_{k}$ by $[\rho(0)]^{-2}=\pi^{2} / 2 N^{2}$ to establish identical scales. We then see that the hardest normal mode eigenvalue for the uncorrelated levels is of order $-2 N^{2} /\left(\pi^{2} \omega_{N}\right)=$ $-(8 / \pi)^{2} N^{2}$ which is comparable to the hardest eigenvalue of $\lambda_{N}=-N^{2}$ obtained for the random matrix ensembles. (The difference is not significant and is largely due to the limitations of the quadratic approximation for the joint probability density.) Significant differences are found in the nature of the soft spectrum. For small values of $k$, the uncorrelated levels reveal a quadratic spectrum, $-2 k^{2}$, which is in sharp contrast to the linear spectrum, $-k N$, of the random matrix ensembles seen in expression (11).

The number variance for a sequence of uncorrelated levels is well known [1]

$$
\Sigma^{2}(L)=L
$$

Here, we wish to proceed in the spirit of the previous section and reproduce this result using the normal models just obtained. Considering the interval $[0, L]$ and repeating the arguments which led to equation (34), we arrive at the expression

$$
\Sigma^{2}(L) \approx \frac{1}{2 N+1} \sum_{k=1}^{N} \frac{1}{\sin ^{2}\left(\phi_{k} / 2\right)} \sin ^{2}\left(L \phi_{k}\right) .
$$

This sum can be performed exactly when $L$ is an integer or a half-integer. For these cases, we find that the number variance is given as

$$
\Sigma^{2}(L) \approx \begin{cases}L & \text { for integer } L \\ L-\frac{1}{2(2 N+1)} & \text { for half-integer } L\end{cases}
$$


for physically interesting values $L<N$. This result agrees with (51) in the large- $N$ limit. For other values of $L$, it is useful to approximate the sum in (52) by an integral as in eqn. (41).

$$
\Sigma^{2}(L) \approx \frac{1}{\pi} \int_{0}^{\pi / 2} \mathrm{~d} x \frac{\sin ^{2}(2 L x)}{x^{2}}=\frac{1}{\pi^{2}}[2 \pi L \operatorname{Si}(2 \pi L)-1+\cos (2 \pi L)],
$$

where $\operatorname{Si}(2 \pi L)$ is the sine integral. For large values of $L$, this leads to the approximation

$$
\Sigma^{2}(L) \approx L-\frac{1}{2 \pi^{3} L} \sin (2 \pi L)-\frac{1}{\pi^{2}},
$$

where we note that the final term of $-1 / \pi^{2}$ is an artifact of having replaced $\sin ^{2} x$ by $x$ in the denominator of (54). Evidently, eqns. (54) and (55) invite comparison with the results of eqns. (41) and (42) obtained for the Gaussian ensembles. We see that the quadratic approximation to the joint probability density also provides a reliable description of the number variance in the large $L$ limit for uncorrelated levels.

\section{Discussion and Conclusions}

We have considered the small amplitude normal modes describing the fluctuations of the eigenvalues of random matrices about their equilibrium positions. In the limit of large matrices, these modes are essentially plane waves. (Recall that the Chebyshev polynomials have the form $U_{2 n}(x)=\cos (2 n+1) x$ and $U_{2 n+1}(x)=\sin (2 n+2) x$ in the limit of large $n$ and fixed $x$.) The mean square amplitude for each mode is inversely proportional to its associated eigenvalue, see eqn. (37). Since these eigenvalues grow monotonically with wave number for both the Gaussian ensembles and for uncorrelated levels, longer wave length fluctuations have a larger amplitude. Thus, the most probable fluctuation in a random matrix spectrum corresponds to a common shift of all eigenvalues with no change in their relative separation, see (12). The next most probable fluctuation is a simple "breathing mode" of the spectrum, see (13).

The properties of the normal mode spectrum provide us with some insight regarding the qualitative behaviour of long-range spectral measures. For uncorrelated levels, the long wave length spectrum is particularly soft with a quadratic spectrum. As a result, $\Sigma^{2}(L)$ is completely dominated by soft modes when $L$ is large. This is seen most easily from eqn. (54). The linear asymptotic behaviour of the number variance for uncorrelated levels is thus seen to be a direct consequence of the quadratic dispersion relation obeyed 
by the soft modes. The situation is qualitatively different in the case of the Gaussian ensembles where all modes obey an exact linear dispersion relation. From eqn. (41) we see that this linearity ensures that $\Sigma^{2}(L)$ must grow logarithmically for large $L$ and that all normal modes contribute democratically to this asymptotic behaviour. Similar linear dispersion relations characterise perfectly elastic solids, and it seems useful to regard the spectral rigidity of random matrices as a consequence of the physical rigidity of a classical one-dimensional array of line charges. Dyson was led to the same conclusion [2]. Although aware of the somewhat arbitrary nature of distinctions between phases in one dimension, he felt it appropriate to call the Coulomb gas (and hence the spectrum of a random matrix) a "crystal". The considerations presented here provide additional support for this designation. In the same spirit, the quadratic dispersion relation for the normal modes of uncorrelated levels leads us to regard them as a gas.

We have emphasised that fluctuations in random matrix spectra are most naturally described as the highly correlated motion of individual eigenvalues, i.e., the normal modes. Given the product form of eqn. (37), it is also clear that these normal modes are statistically independent. If the matrix $D$ of eqn. (43) is calculated in numerical simulations, the statistical errors associated with its eigenvalues will become uncorrelated as the sample size increases. Soft modes (with large amplitudes) and their eigenvalues can thus be determined accurately with relative ease. This fact can be useful in numerical simulations of ensembles which do not readily permit analytic analysis.

\section{Acknowledgements}

We appreciate useful comments on the manuscript by J. Christiansen and K. Splittorff.

\section{References}

[1] T. Guhr, A. Müller-Groeling, and H. A. Weidenmüller: Random-Matrix Theories in Quantum Physics: Common Concepts, Physics Reports 299, 189 (1998).

[2] F. J. Dyson, Statistical Theory of the Energy Levels of Complex Systems, I, J. Math. Phys. 3, 140 (1962); Statistical Theory of the Energy Levels of Complex Systems. II, J. Math. Phys. 3, 157 (1962).

[3] M. L. Mehta, Random Matrices, 2nd Edition, Academic Press (1991). 